Research Paper

\title{
Ocimum gratissmum aqueous extract reduces plasma lipid in hypercholesterol-fed hamsters
} \author{
Te-Jen Lai ${ }^{3,5}$, Jin-Ming Hwang ${ }^{6 凶}$, Je-rYuh Liu $7,8 \bowtie$, Chih-Yang Huang ${ }^{1,9,10 \bowtie ~}$ \\ 1. Graduate Institute of Basic Medical Science, China Medical University, Taichung, Taiwan \\ 2. Department of Laboratory Medicine, Kuang Tien General Hospital, Taichung, Taiwan \\ 3. Institute of Medicine, Chung Shan Medical University, Taichung, Taiwan. \\ 4. Emergency department and center of Hyperbaric Oxygen Therapy, Tungs' Taichung MetroHarbor Hospital, Taichung Taiwan \\ 5. Department of Psychiatry, Chung Shan Medical Hospital, Taichung, Taiwan \\ 6. Department of Applied Chemistry, College of Health Care and Management, Chung Shan Medical University, Taichung, Taiwan \\ 7. Graduate Institute of Cancer Biology, China Medical University, Taichung, Taiwan \\ 8. Center for Molecular Medicine, China Medical University Hospital, Taichung, Taiwan \\ 9. Graduate Institute of Chinese Medical Science, School of Chinese Medicine, China Medical University, Taichung, Taiwan \\ 10. Department of Health and Nutrition Biotechnology, Asia University, Taichung, Taiwan \\ *These authors contributed equally to this work
}

$\triangle$ Corresponding authors: Jer-Yuh Liu PhD., Graduate Institute of Cancer Biology, College of Medical, China Medical University, No 6, Hsueh-Shih Road, Taichung 404, Taiwan. Tel: +886-4-22052121 ext 7932; Fax: +886-4-22347028. E-mail: jyl@mail.cmu.edu.tw. Jin-Ming Hwang, Ph.D. Department of Applied Chemistry, College of Health Care and Management, Chung Shan Medical University, No. 110, Sect. 1, Jianguo N. Road, Taichung 402, Taiwan. Tel: +886-4-24730022 ext. 11875; E-mail: hjm@csmu.edu.tw. Chih-Yang Huang, Graduate Institute of Basic Medical Science, China Medical University, No 91, Hsueh-Shih Road, Taichung 404, Taiwan. Tel.: +886 422053366 ext 3313; Fax: +886 4 22333641. E-mail: cyhuang@mail.cmu.edu.tw.

(C) Ivyspring International Publisher. Reproduction is permitted for personal, noncommercial use, provided that the article is in whole, unmodified, and properly cited. See http://ivyspring.com/terms for terms and conditions.

Received: 2016.06.14; Accepted: 2016.09.01; Published: 2016.10.18

\begin{abstract}
Objectives: Hyperlipidemia is a significant risk factor in the development of atherosclerosis and related diseases which are major health problem in many developed and developing countries that can lead to fatality due to the changes in lifestyle and dietary habits in this modern age.

Methods: In the present study, the Ocimum gratissimum aqueous extract (OGE) was tested for the lowering effect on the serum lipid level of male hamsters on a high-fat (12\%) and high-cholesterol (0.2\%) diet (HFCD).

Results: The results showed that the levels of serum high-density-lipoprotein-cholesterol (HDL-C) low-density-lipoprotein-cholesterol (LDL-C), total cholesterol (TC), and triglycerols (TG) were increased in the HFCD group $(113 \pm 11,259 \pm 87,629 \pm 175$ and $625 \pm 262$, respectively), as compared to the control normal diet group $(51 \pm 8,19 \pm 5,77 \pm 16$ and $101 \pm 44$, respectively). When co-treated with various doses $(10$ and $20 \mathrm{mg} / \mathrm{kg}$ ) of the OGE or rosuvastatin, the rats exhibited the restoration of normal serum LDL-C, TC, and TG levels.
\end{abstract}

Conclusion: Therefore, we suggest that the Ocimum gratissimum aqueous extract may have the potential function of lowering serum lipid in rats.

Key words: Ocimum gratissimum; cholesterol; Hyperlipidemia

\section{Introduction}

Hyperlipidemia is a significant risk factor in the development of atherosclerosis and related diseases which are major health problem in many developed and developing countries that can lead to fatality due to the changes in lifestyle and dietary habits in this modern age $[1,2]$. The research to date on lipid-lowering therapy for intervention is showing this strategy to be effective in reducing the risk of morbidity $[3,4]$, but the cost of lipid-lowering drugs is high, and not everyone is responsive to the treatment.

Natural polyphenols applies to simple molecules (phenolic acids) and highly polymerized compounds 
(tannins) that contribute in the treatment of various diseases of a degenerative nature including hyperlipidemia. Currently there are several thoroughly documented plant-based lipid-lowering polyphenols including those from Ecklonia cava, Ichnocarpus frutescens, Morus alba, Hibiscus sabdariffa, and Red grape [5-9]. Since natural compounds can be used for the prevention of atherosclerosis due to their effect on the reduction of lipid profile in circulating blood and inhibition of LDL-C oxidation $[10,11]$ the search for more forms of herbal remedy is a line of study for the purposes of contributing to a broader understanding of plant-based compounds as well as for increasing locally available treatment options for more geolocations.

Ocimum gratissimum (OG) is an aromatic small shrub that is widely distributed in tropical and warm temperate geolocations. This plant has been prepared in a variety of forms for consumption as food spice or traditional herb in many Asian and African countries since ancient time [12-19]. Recent studies also demonstrate many therapeutic activities of the OG aqueous extract (OGE), including anti-inflammation [17, 20], analgesic and spasmolytic activities [21], antidiarrheal activity [13], antihyperglycemic activity [22], and improvement of phagocytic function [23]. Notably, this extract has also shown potential properties to protect the user from hazardous bio-organisms, such as anthelminthic or insect-repellant properties, antibacterial, antifungal, and antiviral activity [24-28]. Thus, some of more recent researches focus on its role of immune-modulation [29, 30], cancer chemoprevention [31, 34], and prevention of the vulnerability of immune disorder [30]. Moreover, OGE is well documented for its high concentration of phenolic content and effects on free radicals scavenging, lipid peroxidation inhibition, and cytoprotective activity against oxidative stress [20, 32, $33,35,36,37]$. Therefore, in the present study, we evaluated the effects of OGE in preventing hyperlipidemia by using the high fat-cholesterol diet animal model.

\section{Materials and Methods}

\section{Animals and care}

48 male golden Syrian hamsters, aged 4 wks, weighing between 75 and $80 \mathrm{~g}$ were randomly divided into six experimental groups. They were individually housed in metal cages in an air-conditioned room $\left(22 \pm 2{ }^{\circ} \mathrm{C}, 55 \pm 5 \%\right.$ humidity $)$, under a $12 \mathrm{~h}$ light $/ 12 \mathrm{~h}$ dark cycle with free access to food and water. When handled during the 8 week feeding period, all animals used were handled according to the guidelines of the Instituted Animal Care and Use Committee of Chung Shan Medical University (IACUC, CSMC) for the care and use of laboratory animals.

\section{Preparation of diet and supplement}

Experimental hamsters were fed for 8 weeks on a diet containing standard Purina Chow (Purina Mills, Inc.). Chow diet, regarded as a low-fat/cholesterol diet, contains $48.7 \%(\mathrm{w} / \mathrm{w})$ carbohydrate, $23.9 \%$ $(\mathrm{w} / \mathrm{w})$ protein, $5.0 \%(\mathrm{w} / \mathrm{w})$ fat, $5.1 \%(\mathrm{w} / \mathrm{w})$ fiber, and $7.0 \%$ ash (Laboratory Rodent Diet 5001, PMI Nutrition International/Purina Mills LLC, USA).

In order to provoke a hyperlipidemic process, the basal Chow (normal diet) was ground by $12 \%$ $(\mathrm{w} / \mathrm{w})$ coconut oil (high fat diet, HFD) and $0.2 \%$ $(\mathrm{w} / \mathrm{w})$ cholesterol was added to obtain the cholesterol-enriched diet (High Fat/cholesterol diet, HFCD). Diary supplement of OGE at doses of 10 $\mathrm{mg} / \mathrm{kg}$ or $20 \mathrm{mg} / \mathrm{kg}$ per day and $1 \mathrm{mg} / \mathrm{kg}$ rosuvastatin (Crestor, AstraZeneca Inc., Taipei, Taiwan) per day were treated in each experimental group. These Chow containing lipid was pressed, dried at $37^{\circ} \mathrm{C}$ and stored in a dry environment.

Preparation of OGE as indicated in a previous paper [34]. Leaves of Ocimum gratissimum were harvested, washed with distilled water, and then homogenized with distilled water by using polytron. The homogenate was incubated at $95^{\circ} \mathrm{C}$ for 1 hour (h) and then filtered through two layers of gauze. The filtrate was centrifuged to remove insoluble pellets $\left(20,000 \mathrm{~g}\right.$ for $15 \mathrm{~min}$ at $\left.4{ }^{\circ} \mathrm{C}\right)$ and the supernatant (OGE) was collected, lyophilized, and stored at $-70{ }^{\circ} \mathrm{C}$ until use. The content of polyphenol in OGE was analyzed as indicated in a previous paper [35].

\section{Experimental design and data collection}

The dose of OGE used in this study $(10 \mathrm{mg} / \mathrm{Kg}$ or $20 \mathrm{mg} / \mathrm{Kg}$ ) was chosen based on several our earlier studies [36], including a concentration-response study which determined the antioxidant activity of the extract regarding the lipid peroxidation process and studies shown the suitable pharmacological dose in vivo.

Animals were divided into six groups of eight hamsters each and fed for 8 weeks as follows: Group I-standard Purina Chow (normal diet, ND control group); Group II-hamsters fed normal diet mixed with $12 \%$ coconut oil (high fat diet, HFD group); Group III-hamsters fed normal diet mixed with $0.2 \%$ cholesterol and $12 \%$ coconut oil (high fat/cholesterol diet, HFCD group); Group IV- hamsters fed HFCD plus rosuvastatin $1 \mathrm{mg} / \mathrm{Kg}$ body weight per day (HFCD +R group); Group V-hamsters fed HFCD plus OGE $20 \mathrm{mg} / \mathrm{kg}$ body weight per day $(\mathrm{HFCD}+\mathrm{O} 2$ 
group); Group VI-hamsters fed HFCD plus OGE 10 $\mathrm{mg} / \mathrm{kg}$ body weight per day (HFCD+O1 group).

The body weight of each hamster was determined weekly. The food intake of each group was recorded daily and calculated the mean value of each hamster in a week. At the end of 8 weeks, animals were sacrificed by decapitation. Blood samples were collected, and serum separated and stored at $-80{ }^{\circ} \mathrm{C}$ until analysis.

\section{Serum lipid profile estimation}

All biochemical assays were conducted in the clinical laboratories of the Kuang Tien General Hospital, Taichung, Taiwan. The biochemical parameters were measured on a Beckman Coulter Synchron Clinical Systems Analyzer (LX20PRO Autoanalyzer, Beckman Coulter Inc. Taipei, Taiwan). To determine the serum lipid profile, total cholesterol (TC), total triglyceride (TG), low density lipoprotein cholesterol (LDL-C), and high density lipoprotein cholesterol (HDL-C) were measured by Hitachi 7170 autoanalyzer (Hitachi, Tokyo, Japan).

\section{Statistical analysis}

All values are expressed as mean \pm S.D. The significance of differences between the mean of the treated and untreated groups have been calculated by unpaired Student's t-test and P-values less than 0.05 were considered significant.

\section{Results}

\section{Body weight gain, food consumption and observation of hamsters}

The mean body weight $(\mathrm{g})$ and daily food intake (g/hamster) were increased by adequate feeding during the experiment period, as shown in Fig. 1 and Fig. 2. All hamsters in each group survived for the entire length of the study. But in the HFCD control group, the hamsters were visually more lethargic at seven or eight weeks.

There were no differences in mean food intake and body weight among the experimental groups during the 8 weeks of the experiment (Fig. $1 \& 2$ ).

\section{Induction of fatty liver with high fat diet}

At 8 weeks, liver image showed all HFCD groups to have significantly reduced redness compared to the ND and HFD group liver images (Fig. 3). Consistently, relative liver weights of all HFCD groups were also significantly higher (Table 1). Interestingly, the OGE and rosuvastatin supplements did not have an effect on the redness or the weight of the liver.

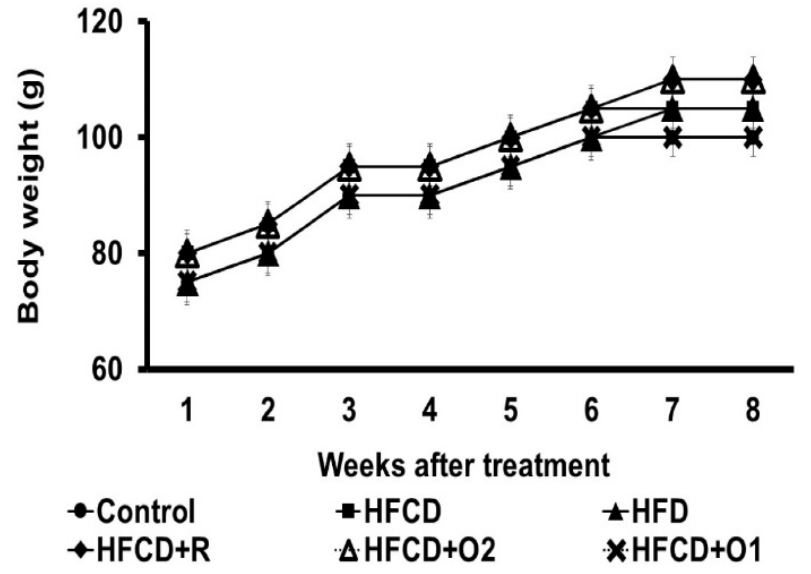

Figure. 1. The change of body weight in hamsters treated with high fat/cholesterol and co-treated with different dose of OGE in eight weeks.

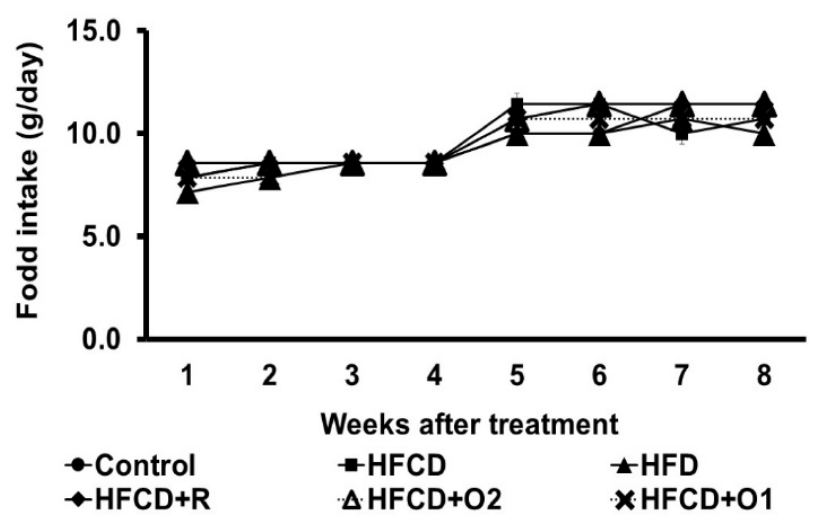

Figure. 2. The change of food intake in hamsters treated with high fat/cholesterol and co-treated with different dose of OGE in eight weeks.

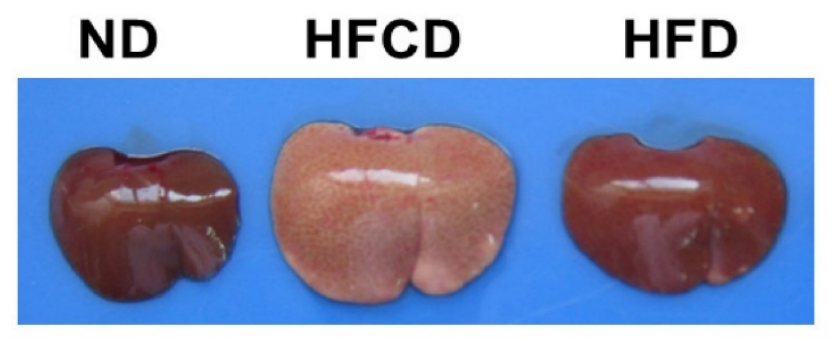

HFCD+R HFCD+O2 HFCD+O1

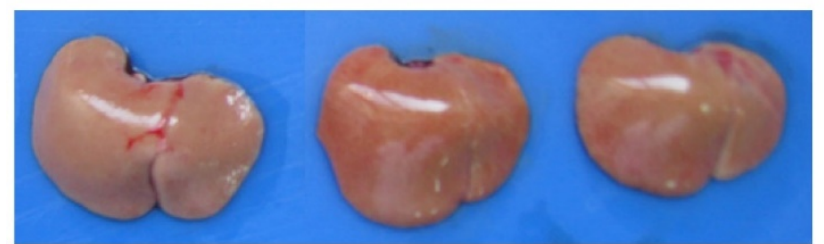

Figure. 3. The image of liver in hamsters treated with high fat/cholesterol and co-treated with different dose of OGE at the end of eight weeks. 
Table 1. The effect of OGE on the change of relative liver weight in hamsters treated high cholesterol diet

\begin{tabular}{llll}
\hline Group & Treatment & No & $\begin{array}{l}\text { Liver related weight } \\
(\%, \text { liver weight/body } \\
\text { weight })\end{array}$ \\
\hline ND & Control & 8 & $2.756 \pm 0.277$ \\
HFCD & Cholesterol + high fat (CF) & 8 & $4.042 \pm 0.521^{*}$ \\
HFD & High fat & 8 & $3.325 \pm 0.265^{*}$, \\
HFCD +R & CF+ Rosurastation 1 mg/kg & 8 & $3.974 \pm 0.337^{*}$ \\
HFCD+O2 & CF+OGE 20 mg/kg & 8 & $3.904 \pm 0.190^{*}$ \\
HFCD+O1 & CF+OGE 10 mg/kg & 8 & $4.014 \pm 0.282^{*}$ \\
*p<0.05 versus control group;, $\mathrm{p}<0.05$ versus Cholesterol and high fat-treated \\
group.
\end{tabular}

\section{Induction of hyperlipidemia with high cholesterol diet}

The levels of plasma TC and TG in ND, HFD, and HFCD groups of hamster at 8 weeks after treatment are reported in Table 2. In comparison with ND group, HFCD caused a marked increase in plasma concentrations of TC and TG by $816 \%$ and $618 \%$, respectively, and both HDL-C and LDL-C increased by more than 2.2 and 13.6 times, respectively. There was no difference between the HDL-C level in the HFD and HFCD groups, while the LDL-C was still far higher in the HFCD in comparison with the HFD hamsters. Moreover, HFCD caused a marked increase in plasma concentrations of TC and TG by $408 \%$ and $353 \%$ as compared to HFD group

Table 2. The effect of OGE on the change of serum lipid in hamster treated high cholesterol diet

\begin{tabular}{llllll}
\hline Treatment & no & HDL-C & LDL-C & TC & TG \\
\hline ND & 8 & $51 \pm 8$ & $19 \pm 5$ & $77 \pm 16$ & $101 \pm 44$ \\
HFCD & 8 & $113 \pm 11^{*}$ & $259 \pm 87^{*}$ & $629 \pm 175^{*}$ & $625 \pm 262^{\#}$ \\
HFD & 8 & $98 \pm 14^{*}$ & $28 \pm 13^{\#}$ & $154 \pm 26^{\#}$ & $177 \pm 64^{*}$ \\
HFCD +R & 8 & $102 \pm 23^{*}$ & $142 \pm 69^{*}, \#$ & $370 \pm 152^{*, \#}$ & $310 \pm 230^{*, \#}$ \\
HFCD+O2 & 8 & $103 \pm 14^{*}$ & $138 \pm 40^{*, \#}$ & $352 \pm 86^{*, \#}$ & $213 \pm 114^{*, \#}$ \\
HFCD+O1 & 8 & $116 \pm 14^{*}$ & $107 \pm 48^{\#}$ & $317 \pm 70^{*, \#}$ & $158 \pm 54^{*}$ \\
\hline
\end{tabular}

In protective testing in hamsters ( 8 weeks application period), a maximum daily dose of OGE $20 \mathrm{mg}$ per $\mathrm{kg}$ body weight was orally administered to hamsters (W.S.).

The data were expressed as mean $\pm S D$. $n=8$. $\# P<0.05$ vs. control group; ${ }^{*} P<0.05$ vs.

High fat and high choesterol group.

\section{Effect of OGE on plasma lipid profile}

After 8 weeks of administration of OGE in lipid enriched-diet fed Hamsters, both plasma TC and TG were decreased by $49.6 \%$ and $74.7 \%$ respectively in HFCD+O1 group, as well as by $44.0 \%$ and $65.9 \%$ respectively in $\mathrm{HFCD}+\mathrm{O} 2$ group, with respect to HFCD group (Table 2). Rosuvastatin, a HMG-CoA reductase inhibitor, was used as reference drug $(1 \mathrm{mg} / \mathrm{Kg}$ body weight) to compare with OGE. The hypolipidemic effect of rosuvastatin in plasma was effective in TC and TG level which were decreased by $41.2 \%$ and $50.4 \%$ (Table 1 ). There were no significant difference in the HDL-C level of all HFCD groups, but
LDL-C in HFCD+O1 and HFCD+O2 groups was decreased by $58.7 \%$ and $46.7 \%$ respectively when compared with HFCD group (Table 2), and the reduction induced by rosuvastatin was $45.1 \%$.

\section{Discussion}

Animal models, such as mouse, rat, golden hamster, guinea pig, rabbit, pigeon and quail are often used for studying hyperlipidemia. Hamster is perhaps the superior model of them not only because their lipid metabolism is similar to that of humans [38, 39], but also that, for hypercholesterolemia studies, the hyperlipidemia content can be easier to maintain by high-fat high-cholesterol induction [40-43].

This study was done to evaluate OGE for its ability to reduce cholesterol and triglyceride in the plasma in hamsters treated with high fat and high cholesterol diets. Table 2 shows that the plasma LDL-C, TC, and TG levels in hamsters, which fed with a high-fat and high-cholesterol diet, were significantly increased. When co-treated with OGE at the same time, the plasma cholesterol levels were restored, similar to the result of anti-cholesterol drug rosuvastatin. We therefore propose the consideration that OGE may have blood lipid lowering effect.

OGE has a large number of simple phenolic compounds and flavonoids, polyphenolic compounds which accounted for $11.1 \%$, including $0.03 \%$ catachin, $0.27 \%$ caffeic acid, $0.37 \%$ epicatechin and $3.27 \%$ rutin [44]. Past studies have indicated that catechins can reduce total cholesterol and LDL, but no significant impact of high-density lipoprotein cholesterol [45]; caffeic acid can inhibit fatty acid synthase, 3-hydroxy-3-methylglutaryl CoA reductase and acyl-CoA:cholesterol acyltransferase activities, while they increased fatty acid beta-oxidation activity and peroxisome proliferator-activated receptors alpha expression in the liver compared to the high-fat group [46]; epicatechin may be an antiaging compound, as evidenced by the improved $\mathrm{db} / \mathrm{db}$ mouse survival and the favorable changes in a variety of age-related biomarkers, including the development of obesity and hyperglycemia [47] and rutin promoted the excretion of fecal sterols, thereby decreasing absorption of dietary cholesterol and lowering the plasma triglyceride levels of diet-induced hypercholesterolemia [48]. Thus, according to these characteristics and comparing to results of the rosuvastatin effects, we suggested that OGE lowering hypercholesterolemia may be mediated through multiple reasons.

\section{Conclusion}

The present study demonstrated that OGE extracted from Ocimum gratissimum may alleviate 
hypercholesterolemia and hypertriglyceridemia induced by the intake of high-fat and high-cholesterol foods. The OGE decreased the levels of TG, total cholesterol, and LDL-C, and improved HDL-C levels. Although the metabolic mechanisms are not identical between the four flavonoids, the pharmacological effects of OGE should be further investigated in humans. These findings may contribute to the treatment of obesity- and hyperlipidemia-associated diseases in humans.

\section{Acknowledgements}

This work was supported by grants from the Ministry of Science and Technology, Republic of China (MOST 104-2320-B-039-032), as well as in part by Taiwan Ministry of Health and Welfare Clinical Trial and Research Center of Excellence (MOHW105-TDU-B-212-133019).

\section{Competing Interests}

The authors have declared that no competing interest exists.

\section{References}

1. Schwartz CJ, Valente AJ, Sprague EA. A modern view of atherogenesis. Am J Cardiol. 1993; 71(6): 9B-14B.

2. Mannu GS, Zaman MJ, Gupta A, et al. Evidence of lifestyle modification in the management of hypercholesterolemia. Curr Cardiol Rev. 2013; 9(1): 2-14.

3. Blankenhorn DH, Hodis HN. Treating serum lipid abnormalities in highpriority patients. Postgrad Med. 1991; 89(1): 81-82, 87-90, 93-96.

4. Okere AN, Serra C. Evaluation of the Potential Role of Alirocumab in the Management of Hypercholesterolemia in Patients with High-Risk Cardiovascular Disease. Pharmacotherapy. 2015; 35(8): 771-779.

5. Hyeon CS, Seong HK, Yongju P, et al. Effects of 12 week oral supplementation Ecklonia cava polyphenols on anthropometric and blood lipid parameters in of overweight Korean individuals: a double-blind randomized clinical trial. Phytother Res. 2012; 26(3): 363-368.

6. Chidambaram T, Kumarappan T, Rao N. Polyphenolic extract of Ichnocarpus frutescens modifies hyperlipidemia status in diabetic rats. J Cell Mol Biol. 2007; 6(2): 175-187.

7. Mon YY, Chiung HP, Kuei CC, et al. The hypolipidemic effect of Hibiscus sabdariffa polyphenols via inhibiting lipogenesis and promoting hepatic lipid clearance. J Agric Food Chem. 2010; 58: 850-859.

8. Mon $\mathrm{YY}$, Chien $\mathrm{NH}$, Kuei $\mathrm{CC}$, et al. Mulberry leaf polyphenols possess antiatherogenesis effect via inhibiting LDL oxidation and foam cell formation. J Agric Food Chem. 2011; 59: 1985-1995.

9. Alberto D, Carlos FH, Francisca C, et al. Red grape juice polyphenols alter cholesterol homeostasis and increase LDL receptor activity in human cells in vitro. J Nut. 2006; 136(7): 1766-1773.

10. Chi F, Mao T, Simon J. Analysis of antioxidant as a therapeutic agent for atherosclerosis. Curr Pharm Anal, 2006; 2 :369-384.

11. Bahmani M, Mirhoseini M, Shirzad H, et al. A review on promising natural agents effective on hyperlipidemia. J Evid Based Complementary Altern Med. 2015; 20(3): 228-238.

12. Onajobi FD. Smooth muscle contracting lipid-soluble principles in chromatographic fractions of Ocimum gratissimum. J Ethnopharmacol. 1986; 18(1): 3-11.

13. Ilori M, Sheteolu AO, Omonigbehin EA, et al. Antidiarrhoeal activities of Ocimum gratissimum (Lamiaceae). J Diarrhoeal Dis Res. 1996; 14(4): 283-285.

14. Fandohan P, Gnonlonfin B, Laleye A, et al. Toxicity and gastric tolerance of essential oils from Cymbopogon citratus, Ocimum gratissimum and Ocimum basilicum in Wistar rats. Food Chem Toxicol. 2008; 46(7): 2493-2497.

15. Fabrizio DM, Ilaria B, Andrea Galimberti, et al. A comparative study of different DNA barcoding markers for the identification of some members of Lamiacaea. Food Research International. 2011; 44(3): 693-702.

16. Santanu K M, Subhankari P C, Subhasis D, et al. Methanol extract of Ocimum gratissimum protects murine peritoneal macrophages from nicotine toxicity by decreasing free radical generation, lipid and protein damage and enhances antioxidant protection. Oxid Med Cell Longev. 2009; 2(4): 222-230.

17. Lin CC, Lin JK, Chang CH. Evaluation of hepatoprotective effects of "ChhitChan-Than" from Taiwan. Pharmaceutical Biology. 1995; 33(2): 139-143.

18. Ouyang $X$, Liuxin $W$, Yingming $P$, et al. Antioxidant properties and chemical constituents of ethanolic extract and its fractions of Ocimum gratissimum. Medicinal Chemistry Research. 2013; 22(3): 1124-1130.

19. Okoli CO, Ezike AC, Agwagah OC, et al. Anticonvulsant and anxiolytic evaluation of leaf extracts of Ocimum gratissimum, a culinary herb. $\mathrm{P}$ harmacognosy Res. 2010; 2(1): 36-40

20. Lee MI, Chen HM, Tzang BS, et al. Ocimum gratissimum Aqueous Extract Protects H9c2 Myocardiac Cells from H2O2-Induced Cell Apoptosis through Akt Signalling. Evid Based Complement Alternat Med. 2011; 2011: 578060.

21. Aziba PI, Bass D, Elegbe Y, et al. Pharmacological investigation of Ocimum gratissimum in rodents. Phytother Res. 1999; 13(5): 427-429.

22. Aguiyi JC, Obi CI, Gang SS, et al. Hypoglycaemic activity of Ocimum gratissimum in rats. Fitoterapia. 2000; 71(4): 444-6.

23. Atal CK, Sharma ML, Kaul A, et al. Immunomodulating agents of plant origin. I: Preliminary screening. J Ethnopharmaco. 1986; 18(2): 133-141.

24. Dubey NK, Tiwari TN, Mandin D, et al. Antifungal properties of Ocimum gratissimum essential oil (ethyl cinnamate chemotype). Fitoterapia. 2000; 71(5): 567-569

25. Pessoa LM, Morais SM, Bevilaqua CM, et al. Anthelmintic activity of essential oil of Ocimum gratissimum Linn. and eugenol against Haemonchus contortus. Vet Parasitol. 2002; 109(1-2): 59-63.

26. Ayisi NK, Nyadedzor C. Comparative in vitro effects of AZT and extracts of Ocimum gratissimum, Ficus polita, Clausena anisata, Alchornea cordifolia, and Elaeophorbia drupifera against HIV-1 and HIV-2 infections. Antiviral Res. 2003; 58(1): 25-33.

27. Martin KW, Ernst E. Herbal medicines for treatment of bacterial infections: a review of controlled clinical trials. J Antimicrob Chemother. 2003; 51(2): 241-246.

28. Silva MR, Oliveira JG Jr, Fernandes OF, et al. Antifungal activity of Ocimum gratissimum towards dermatophytes. Mycoses. 2005; 48(3): 172-175.

29. Costa RS, Carneiro TC, Cerqueira-Lima AT, et al. Ocimum gratissimum Linn. And rosmarinic acid, attenuate eosinophilic airway inflammation in an experimental model of respiratory allergy to Blomia tropicalis. Int Immunopharmacol. 2012; 13(1): 126-134.

30. Mahapatra SK, Chakraborty SP, Roy S. Immunomodulatory role of Ocimum gratissimum and ascorbic acid against nicotine-induced murine peritoneal macrophages in vitro. Oxid Med Cell Longev. 2011. 2011: 734319.

31. Nangia-Makker P, Tait L, Shekhar MP, et al. Inhibition of breast tumor growth and angiogenesis by a medicinal herb: Ocimum gratissimum. Int J Cancer. 2007;121(4): 884-894.

32. Chiu YW, Lo HJ, Huang HY et al. The antioxidant and cytoprotective activity of Ocimum gratissimum extracts against hydrogen peroxide-induced toxicity in human HepG2 cells. JFDA. 2013; 21(3): 253-260.x

33. Li PC, Chiu YW, Lin YM, et al. Herbal Supplement Ameliorates Cardiac Hypertrophy in Rats with CCl4-Induced Liver Cirrhosis. Evid Based Complement Alternat Med. 2012. 2012: 139045.

34. Chen HM, Lee MJ, Kuo CY, et al. Ocimum gratissimum Aqueous Extract Induces Apoptotic Signalling in Lung Adenocarcinoma Cell A549. Evid Based Complement Alternat Med. 2011. 2011: 739093.

35. Chiu CC, Huang CY, Chen TY, et al. Beneficial Effects of Ocimum gratissimum Aqueous Extract on Rats with CCl4-Induced Acute Liver Injury. Evid Based Complement Alternat Med. 2012. 2012: 736752

36. Chiu YW, Chao PY, Tsai CC, et al. Ocimum gratissimum is effective in prevention against liver fibrosis in vivo and in vitro. Am J Chin Med. 2014; 42(4): 833-852.

37. Lin YC, Cheng KM, Huang HY, et al. Hepatoprotective activity of Chhit-ChanThan extract powder against carbon tetrachloride-induced liver injury in rats. JFDA. 2014; 22(2): 220-229.

38. Liu XM, Wu FH. Comparison of animal models of hyperlipidemia. Zhong Xi Yi Jie He Xue Bao. 2004; 2: 132-134.

39. Zhang Z, Wang $\mathrm{H}$, Jiao $\mathrm{R}$, et al. Choosing hamsters but not rats as a model for studying plasma cholesterol-lowering activity of functional foods. Mol Nutr Food Res. 2009; 53: 921-930

40. Sullivan MP, Cerda JJ, Robbins FL, et al. The gerbil, hamster, and guinea pig as rodent models for hyperlipidemia. Lab Anim Sci. 1993; 43:575-578.

41. Gao Y, Li K, Tang S, et al. Study on animal models for hyperlipidemia. Wei Sheng Yan Jiu. 2002; 31: 97-99.

42. Lee CL, Tsai TY, Wang JJ, Pan T.M. In vivo hypolipidemic effects and safety of low dosage Monascus powder in a hamster model of hyperlipidemia. Appl Microbiol Biotechnol. 2006; 70: 533-540.

43. Lee CL, Hung H., Wang JJ. Red mold dioscorea has greater hypolipidemic and antiatherosclerotic effect than traditional red mold rice and unfermented dioscorea in hamsters. J Agric Food Chem. 2007; 55(17): 7162-7169.

44. Chen $\mathrm{YH}$, Chiu $\mathrm{YW}$, Shyu JC, et al. Protective effects of Ocimum gratissimum polyphenol extract on carbon tetrachloride-induced liver fibrosis in rats. Chin J Physiol. 2015; 58(1): 55-63

45. Kim A, Chiu A, Barone MK, et al. Green tea catechins decrease total and low-density lipoprotein cholesterol: a systematic review and meta-analysis. J Am Diet Assoc. 2011; 111(11): 1720-1729. 
46. Cho AS1, Jeon SM, Kim MJ, et al. Chlorogenic acid exhibits anti-obesity property and improves lipid metabolism in high-fat diet-induced-obese mice. Food Chem Toxicol. 2010; 48(3): 937-943.

47. Si H1, Fu Z, Babu PV, et al. Dietary epicatechin promotes survival of obese diabetic mice and Drosophila melanogaster. J Nutr. 2011; 141(6): 1095-1100.

48. Kanashiro A, Andrade DC, Kabeya LM, et al. Modulatory effects of rutin on biochemical and hematological parameters in hypercholesterolemic Golden Syrian hamsters. An Acad Bras Cienc. 2009; 81(1): 67-72. 